\title{
Preoperative Factors Associated with Press Ganey Patient Satisfaction Scores after Anterior Cruciate Ligament Reconstruction
}

\author{
Gregory T. Perraut, MD ${ }^{1}$ Tina Zhang, MD ${ }^{2}$ Tristan B. Weir, MD ${ }^{2}$ Matheus B. Schneider, BS 20 \\ Ali Aneizi, MD ${ }^{2}$ Natalie L. Leong, MD $^{2}$ Jonathan D. Packer, MD $^{2}$ Sean J. Meredith, MD ${ }^{2}$ \\ R. Frank Henn III, MD20
}

\footnotetext{
${ }^{1}$ Department of Orthopedics, Georgetown University Medical Center, Washington, District of Columbia

2 Department of Orthopedics, University of Maryland School of Medicine, Baltimore, Maryland
}

Address for correspondence R. Frank Henn III, MD, Department of Orthopedics, University of Maryland Rehabilitation and Orthopedic Institute, 2200 Kernan Drive, Baltimore, MD 21207

(e-mail: fhenn@som.umaryland.edu).

J Knee Surg 2023;36:673-681.

\author{
Abstract \\ Keywords \\ - anterior cruciate \\ ligament \\ reconstruction \\ - $\mathrm{ACL}$ \\ - Press Ganey \\ - satisfaction \\ - ACLR
}

Patient satisfaction is increasingly used as a metric to evaluate the quality of healthcare services and to determine hospital and physician compensation. The aim of this study was to identify preoperative factors associated with Press Ganey Ambulatory Surgery (PGAS) satisfaction scores, and to evaluate the effect of each PGAS domain score on the total PGAS score variability in patients undergoing anterior cruciate ligament reconstruction (ACLR). A review of a Press Ganey (PG) database at a single center was performed for patients undergoing ACLR between 2015 and 2019. Ninety-nine patients completed the PGAS survey and 54 also completed preoperative demographic and patient-reported outcome measures (PROMs) for an orthopaedic registry. PGAS scores were calculated and bivariate analysis was performed. Multivariable linear regression determined the effect of each of the six PGAS domains on the total PGAS score variability. In the total cohort of 99 patients, no factors were significantly associated with the total PGAS score or any domain scores. For the 54 patients who also participated in the orthopaedic registry, none of the preoperative PROMs were significantly correlated with total PGAS score. However, having a college degree (89 vs. 95 or 97 points; $p=0.02$ ) and continuous femoral nerve catheter ( 92 vs. 100 points; $p=0.04$ ) was associated with lower personal issue domain scores, while patients with a greater number of prior surgeries had worse registration domain scores $(\rho=-0.27$; $p=0.049$ ). For the entire cohort, the registration and facility domains contributed the most variability to the total PGAS score, while the physician domain contributed the least. Few preoperative factors are associated with PGAS scores, and total PGAS scores do not significantly correlate with baseline PROMs. Surgeons may have limited ability to improve their PGAS scores given most of the variability in total scores stems from systemic aspects of the patient experience. received

August 7, 2021

accepted after revision

November 16, 2021

article published online

January 3, 2022 (c) 2022. Thieme. All rights reserved.

Thieme Medical Publishers, Inc., 333 Seventh Avenue, 18th Floor, New York, NY 10001, USA
DOI https://doi.org/

10.1055/s-0041-1741394. ISSN 1538-8506. 
Under the Hospital Value-Based Purchasing Program, established by the Patient Protection and Affordable Care Act of 2010, the Centers for Medicare and Medicaid Services issues incentive payments to healthcare institutions based on various quality domains, including the patient experience. ${ }^{1,2}$ As such, patient satisfaction scores have been increasingly used by organizations to evaluate physician performance and determine compensation. ${ }^{3}$ However, satisfaction has been shown to be influenced by factors such as age, education, spirituality, and overall physical and mental health. ${ }^{1,2}$ Thus, identifying the baseline patient demographics, surgical characteristics, and patient-reported outcome measures (PROMs) that are associated with worse patient satisfaction may help orthopaedic surgeons maximize patient care and provider reimbursement.

Press Ganey (PG) Associates LLC (Elkridge, MD) is a private company utilized by over 10,000 healthcare organizations and more than $50 \%$ of hospitals to administer patient satisfaction surveys. ${ }^{4}$ PG satisfactions scores have been investigated in prior orthopaedic literature, but those studies have been limited to outpatient clinic or inpatient encounters for total joint arthroplasty, spine, and hand surgeries. ${ }^{1-3,5-8}$ The only studies evaluating PG scores in patients undergoing knee surgery have been in patients undergoing total knee arthroplasty. ${ }^{1,7,9}$ These studies have shown poor correlations between legacy outcome measures and PG scores, questioning the utility of such patient satisfaction metrics. There is a lack of data, however, on the factors associated with PG scores in patients undergoing anterior cruciate ligament reconstruction (ACLR) in the ambulatory surgical setting.

The purpose of this study was to identify the factors associated with PG scores in patients undergoing ACLR. First, we hypothesized that certain patient and surgical factors would correlate with worse PG scores. Second, we further hypothesized that worse baseline PROMs would be associated with worse PG scores. Finally, we hypothesized that certain PG domains would contribute more to the variability of the total score than other domains.

\section{Methods}

Patients undergoing primary or revision ACLR at a single urban center between June 2015 and August 2019 were identified retrospectively under approval of the institutional review board. At our institution, all adult patients $(\geq 18$ years) received the PG Ambulatory Surgery (PGAS) survey within 48 hours to 6 weeks postoperatively by mail or e-mail. During the study period, nine surgeons performed 829 ACLR procedures and a total of 99 patients completed the PGAS survey (11.9\%).

The PGAS survey includes 35 questions pertaining to the patient experience, divided into six domains: (1) registration, (2) facility, (3) nursing, (4) physician, (5) personal issues, and (6) overall assessment. The PGAS survey is based on a 5-point Likert scale with responses of very poor, poor, fair, good, and very good, which are then converted to a 0 to 100 scale. The mean scores for questions within a domain make up the domain score, which are averaged to calculate the PGAS total score. A score of 100 for each domain and the total score indicates perfect satisfaction. ${ }^{10}$ In addition to the PGAS scores, variables from the PG database included age, sex, ethnicity, race, insurance type, surgeon, surgical characteristics, survey type (mail versus e-mail), and the survey completion date.

Four surgeons also participated in a prospective orthopaedic registry utilizing the Research Electronic Data Capture (REDCap) data collection system, and 54 of the 99 patients were enrolled in the registry. ${ }^{11,12}$ Variables obtained from the registry included body mass index, the number of any prior surgeries, comorbid conditions, education level, employment status, income, marital status, smoking status, alcohol or drug use, preoperative narcotic use, and regional anesthesia type. Multiple baseline PROMs were obtained from the registry. Patients completed six domains of the Patient Reported Outcomes Measurement Information System (PROMIS; physical function, pain interference, fatigue, social satisfaction, anxiety, and depression) computeradaptive test (CT). Knee and total body pain were assessed with the numeric pain scale. Expectations of surgery were assessed with the expectations domain of the musculoskeletal outcomes data evaluation and management system. Patient physical activity was measured with the Tegner activity scale and Marx knee activity rating scale. Kneespecific outcome measures included the International Knee Documentation Committee (IKDC) Subjective Knee Evaluation Form.

Continuous data was reported as means and standard deviations, while categorical data was reported as frequencies and percentages. Wilcoxon signed-rank tests and Kruskal-Wallis tests were used for the analysis of continuous variables, and chi-squared tests for categorical variables. The Bonferroni correction was used for any posthoc pairwise comparisons on significant findings in categorical variables with more than two levels. The Spearman rank correlation coefficient was used to determine the correlations between PGAS scores and other continuous variables. Multivariable analysis with stepwise backward linear regression was implemented to evaluate total PGAS score as a function of the six domains of the PGAS survey. The standardized parameter estimates and the incremental impact of each parameter to the model's adjusted $\mathrm{R}^{2}$ value were used to determine relative importance of each PGAS domain. The incremental impact of each parameter was calculated by determining the change in the adjusted $\mathrm{R}^{2}$ when that parameter was the last independent variable entered into the model. JMP Pro, Version 13 software (JMP, Version 13. SAS Institute Inc., Cary, NC) was used for all analyses, and a $p$ value $<0.05$ indicated statistical significance. All $p$-values were two-tailed.

\section{Results}

Fifty-four of the 99 patients (54\%) included in the study completed the PGAS survey and also participated in the orthopaedic registry (registry group), while 45 of the 99 patients (45\%) only completed the PGAS survey (PG only 
Table 1 Baseline characteristics of the PG only versus registry group ${ }^{a}$

\begin{tabular}{|c|c|c|c|}
\hline Factors & PG only $(n=45)$ & Registry $(n=54)$ & $p$-Value \\
\hline Survey days postop & $24.6 \pm 28.4$ & $19.1 \pm 19.8$ & 0.79 \\
\hline Age, $y$ & $29.0 \pm 12.4$ & $29.1 \pm 12.4$ & 0.83 \\
\hline Male & $26(58)$ & $26(48)$ & 0.34 \\
\hline \multicolumn{4}{|l|}{ Race } \\
\hline White & $32(71)$ & $35(66)$ & \multirow[t]{3}{*}{0.83} \\
\hline Black & $5(11)$ & $6(11)$ & \\
\hline Other & $8(18)$ & $12(23)$ & \\
\hline \multicolumn{4}{|l|}{ Insurance } \\
\hline Commercial & $38(84)$ & $49(91)$ & \multirow[t]{2}{*}{0.58} \\
\hline Noncommercial & $7(16)$ & $6(9)$ & \\
\hline Primary ACLR & $40(89)$ & $48(89)$ & 0.99 \\
\hline \multicolumn{4}{|l|}{ Meniscus tear } \\
\hline Medial only & $9(20)$ & $8(15)$ & 0.50 \\
\hline Lateral only & $13(29)$ & $17(31)$ & 0.78 \\
\hline Both & $4(9)$ & $15(28)$ & 0.018 \\
\hline Any & $26(58)$ & $40(74)$ & 0.004 \\
\hline
\end{tabular}

Abbreviations: ACLR, anterior cruciate ligament reconstruction; PG, Press Ganey; SD, standard deviation.

Note: Bold indicates $p<0.05$.

${ }^{a}$ Values are presented as No. (\%) or mean \pm SD.

group) (-Table 1). There were no significant differences in baseline characteristics or PG scores between the two groups, aside from the number of meniscus tears ( - Tables 1 and 2).

- Table 3 shows PGAS scores by preoperative variables for the total cohort of 99 patients. No significant associations were observed.

For the 54 patients in the registry group, - Table 4 shows differences in PGAS scores by additional patient and surgical characteristics captured in the orthopaedic registry. Having a college degree was associated with significantly lower personal issue scores compared with high school graduates $(p=0.047)$ and those without high school degrees $(p=0.03)$. Additionally, patients who received a continuous femoral nerve catheter also had lower personal issue scores $(p=0.04)$. Having more prior procedures was correlated with lower registration scores $(p=0.049)$. None of the baseline PROMs had significant strong correlations with PGAS scores, but baseline PROMIS anxiety was significantly weakly correlated with the overall assessment domain score $(p=0.04)$ ( - Table 5).

The multivariable linear regression model for the total PGAS score is shown in - Table 6. All six PGAS domains were significant independent predictors of the total score, and the registration domain had the highest standardized parameter estimate and had the greatest impact on the model's adjusted $R^{2}$ value. The physician domain had the lowest standardized parameter estimate and impact on the model's adjusted $R^{2}$ value.

Table 2 Distribution of Press Ganey ambulatory surgery scores ${ }^{a}$

\begin{tabular}{|l|l|l|l|l|}
\hline Scores & Total $(\boldsymbol{n}=\mathbf{9 9})$ & PG only $\mathbf{b}^{\mathbf{b}}(\boldsymbol{n}=\mathbf{4 5})$ & Registry $^{\mathbf{b}}(\boldsymbol{n}=\mathbf{5 4})$ & $\boldsymbol{p}^{\text {Value }}$ \\
\hline Total & $92.0 \pm 9.6$ & $90.1 \pm 11.6$ & $93.6 \pm 7.3$ & 0.26 \\
\hline Facility & $86.2 \pm 13.2$ & $85.0 \pm 13.6$ & $87.2 \pm 12.9$ & 0.40 \\
\hline Registration & $89.3 \pm 13.7$ & $86.2 \pm 16.0$ & $91.9 \pm 10.8$ & 0.10 \\
\hline Nursing & $94.0 \pm 12.3$ & $91.2 \pm 16.6$ & $96.3 \pm 6.4$ & 0.49 \\
\hline Physician & $96.3 \pm 8.2$ & $95.8 \pm 8.9$ & $96.8 \pm 7.7$ & 0.74 \\
\hline Personal issue & $90.6 \pm 12.9$ & $88.4 \pm 15.3$ & $92.4 \pm 10.4$ & 0.37 \\
\hline Overall assessment & $95.6 \pm 10.9$ & $93.7 \pm 13.7$ & $97.2 \pm 7.6$ & 0.13 \\
\hline
\end{tabular}

Abbreviations: PG, Press Ganey; SD, standard deviation.

${ }^{a}$ Values are presented as mean \pm SD.

'The PG only group consists of patients who were not included in the registry, while the registry patients completed additional questionnaires. 


\begin{tabular}{|c|c|c|c|c|c|c|c|c|c|c|c|c|c|c|c|c|c|c|c|c|c|c|c|c|c|c|}
\hline & $\begin{array}{l}\frac{0}{2} \\
\frac{\pi}{n} \\
\frac{1}{2}\end{array}$ & $\begin{array}{l}\hat{0} \\
0 \\
0\end{array}$ & $\begin{array}{l}\overline{6} \\
0\end{array}$ & & 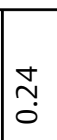 & & & $\begin{array}{l}0 \\
0 \\
0\end{array}$ & & & & 䚄 & & & & f̊. & & $\begin{array}{l}\stackrel{9}{~} \\
0\end{array}$ & & & $\begin{array}{l}\stackrel{\mathscr{L}}{0} \\
\stackrel{0}{0}\end{array}$ & & & $\stackrel{n}{m}$ & & \\
\hline $\begin{array}{l}\overline{\bar{\pi}} \\
\frac{0}{0} \\
0 \\
0\end{array}$ & ڤั & $\begin{array}{l}0 \\
0 \\
0 \\
1\end{array}$ & \begin{tabular}{|l|} 
\\
\\
0 \\
0 \\
1
\end{tabular} & & $\mid \begin{array}{l}\sim \\
+ \\
\tilde{n} \\
\Omega\end{array}$ & $\begin{array}{l}a \\
+ \\
\hat{\sigma}\end{array}$ & & $\begin{array}{l}\infty \\
+1 \\
\hat{\sigma} \\
\end{array}$ & $\begin{array}{l}\bar{N} \\
+ \\
\bar{\sigma}\end{array}$ & $\begin{array}{l}= \\
\text { ㅍ } \\
\text { nू }\end{array}$ & & $\begin{array}{l}F \\
\bar{H} \\
\tilde{\sigma}\end{array}$ & $\begin{array}{l}a \\
+ \\
\hat{\sigma}\end{array}$ & $\begin{array}{l}0 \\
\text { H } \\
8 \\
0 \\
\end{array}$ & & $\begin{array}{l}\sigma \\
+ \\
\mathscr{L} \\
\sigma\end{array}$ & 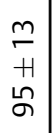 & 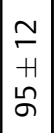 & & $\begin{array}{l}\sigma \\
+1 \\
\varnothing \\
\varnothing\end{array}$ & 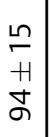 & $\begin{array}{l}0 \\
+1 \\
\Omega\end{array}$ & & $\begin{array}{l}= \\
+ \\
\ddot{8}\end{array}$ & $\begin{array}{l}- \\
+ \\
\infty \\
\sigma\end{array}$ & \\
\hline . & 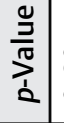 & $\begin{array}{l}0 \\
0 \\
0 \\
0\end{array}$ & $\begin{array}{l}0 \\
0 \\
0 \\
\end{array}$ & & $\stackrel{0}{\check{0}}$ & & & হ̄. & & & & \begin{tabular}{l}
0 \\
\multirow{1}{0}{} \\
0
\end{tabular} & & & & 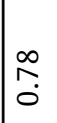 & & $\begin{array}{l}0 \\
\tilde{n} \\
0\end{array}$ & & & $\begin{array}{l}g \\
\stackrel{9}{0}\end{array}$ & & & @̊. & & \\
\hline 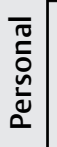 & $\begin{array}{l}0 \\
\vdots \\
\tilde{u}\end{array}$ & $\begin{array}{l}n \\
0 \\
\dot{0} \\
1\end{array}$ & $\begin{array}{l}n \\
\circ \\
0 \\
0\end{array}$ & & $\mid \begin{array}{l}m \\
+ \\
+ \\
\infty \\
\infty\end{array}$ & $\mid \begin{array}{l}m \\
+ \\
\sigma \\
\sigma\end{array}$ & & $\mid \begin{array}{l}\simeq \\
+1 \\
\sigma\end{array}$ & 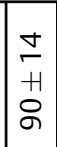 & $\begin{array}{l}6 \\
+ \\
+1 \\
\infty \\
\infty\end{array}$ & & $\begin{array}{l}m \\
\stackrel{m}{H} \\
\stackrel{\circ}{\circ}\end{array}$ & 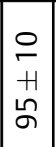 & $\begin{array}{l}\text { in } \\
+1 \\
\infty \\
\infty\end{array}$ & & $\begin{array}{l} \pm \\
+ \\
\stackrel{+}{\circ} \\
\varnothing\end{array}$ & $\begin{array}{l}\simeq \\
+ \\
\bar{\sigma}\end{array}$ & 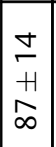 & & $\begin{array}{l}\simeq \\
+1 \\
\tilde{n} \\
\sigma\end{array}$ & $\begin{array}{l}\simeq \\
+ \\
\bar{\sigma}\end{array}$ & $\begin{array}{l}\sigma \\
H \\
\text { G }\end{array}$ & & & $\begin{array}{l}\frac{m}{7} \\
\bar{\sigma} \\
\sigma\end{array}$ & \\
\hline 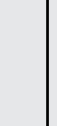 & $\begin{array}{l}\frac{9}{3} \\
\frac{2}{\pi} \\
\frac{1}{2}\end{array}$ & $\mid \begin{array}{l}\infty \\
0 \\
0 \\
0\end{array}$ & 占 & & $\stackrel{\substack{m \\
o}}{0}$ & & & 贻 & & & & פ్ & & & & $\begin{array}{l}\text { ำ } \\
\text { กุ. }\end{array}$ & . & 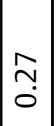 & & & $\begin{array}{l}\text { ใ̊ } \\
0 \\
0\end{array}$ & & & $\stackrel{\text { fo }}{\circ}$ & & \\
\hline : & 竞 & $\begin{array}{l}0 \\
0 \\
0\end{array}$ & $\begin{array}{l}0 \\
0 \\
0 \\
1\end{array}$ & & $\begin{array}{l}\infty \\
+1 \\
6 \\
\swarrow\end{array}$ & $\begin{array}{l}\infty \\
+1 \\
\hat{\sigma}\end{array}$ & & $\begin{array}{l}0 \\
+ \\
\hat{n} \\
\end{array}$ & $\begin{array}{l}F \\
\bar{H} \\
\tilde{m}\end{array}$ & $\begin{array}{l}\circ \\
+ \\
+ \\
\text { ㅁ } \\
\Omega\end{array}$ & & $\begin{array}{l}\infty \\
+ \\
+ \\
\mathscr{Q}\end{array}$ & $\begin{array}{l}a \\
H \\
b \\
\sigma\end{array}$ & $\mid$\begin{tabular}{l|}
$m$ \\
+ \\
$\infty$ \\
$\infty$
\end{tabular} & & \begin{tabular}{l|}
$\infty$ \\
+1 \\
0 \\
$\sigma$
\end{tabular} & $\begin{array}{l}a \\
+1 \\
\hat{\sigma}\end{array}$ & $\begin{array}{l}0 \\
+ \\
\infty \\
\sigma \\
\end{array}$ & & 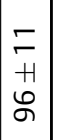 & $\begin{array}{l}\infty \\
+ \\
\emptyset \\
\sigma\end{array}$ & 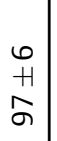 & & & $\begin{array}{l}r \\
+ \\
\infty \\
\sigma\end{array}$ & \\
\hline & 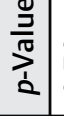 & \begin{tabular}{c}
0 \\
\multirow{2}{0}{} \\
0
\end{tabular} & $\mid \begin{array}{l}0 \\
0 \\
0 \\
0\end{array}$ & & 六. & & & $\mp$ & & & & $\begin{array}{l}+ \\
6 \\
0\end{array}$ & & & & $\begin{array}{l}\hat{\sigma} \\
0\end{array}$ & & $\begin{array}{l}\text { ț } \\
0 \\
0\end{array}$ & & & $\bar{\sigma}$ & & & $\begin{array}{l}\stackrel{L}{\infty} \\
\infty \\
0\end{array}$ & & \\
\hline 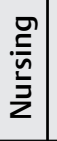 & $\begin{array}{l}0 \\
\vdots \\
\sim \\
\sim\end{array}$ & $\begin{array}{l}0 \\
0 \\
0 \\
1\end{array}$ & \begin{tabular}{|c|}
0 \\
0 \\
0 \\
1
\end{tabular} & & $\begin{array}{l}\nabla \\
+ \\
m \\
\\
\end{array}$ & $\begin{array}{l}\circ \\
\\
+1 \\
\mathscr{8} \\
\sigma\end{array}$ & & $\begin{array}{l}\sigma \\
+ \\
H \\
2 \\
\sigma \\
\end{array}$ & $\begin{array}{l}\tilde{N} \\
+ \\
\sim \\
\infty\end{array}$ & $\begin{array}{l}\vec{J} \\
\bar{H} \\
\tilde{\sigma} \\
\tilde{\sigma}\end{array}$ & & $\begin{array}{l}m \\
\stackrel{n}{+} \\
\dot{5} \\
\end{array}$ & $\begin{array}{l}0 \\
+ \\
+ \\
\sigma \\
\sigma \\
\end{array}$ & $\begin{array}{l}N \\
+1 \\
\sigma \\
\sigma\end{array}$ & & 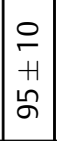 & 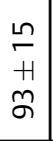 & $\begin{array}{l}+ \\
+ \\
\infty \\
\sigma \\
\end{array}$ & & \begin{tabular}{l|}
$\circ$ \\
\\
+1 \\
5 \\
$\sigma$
\end{tabular} & $\begin{array}{l}\stackrel{2}{\tau} \\
+ \\
\dot{\sigma}\end{array}$ & 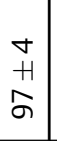 & & & $\begin{array}{l}\text { i } \\
+ \\
\mathscr{\sigma}\end{array}$ & \\
\hline ธ & $\begin{array}{l}\frac{0}{0} \\
\frac{\partial}{50} \\
\vdots \\
\vdots \\
0\end{array}$ & $\begin{array}{l}0 \\
\\
0\end{array}$ & $\begin{array}{l}\overline{0} \\
0 \\
0\end{array}$ & & $\begin{array}{l}0 \\
\vdots \\
0 \\
\end{array}$ & & & 음. & & & & פ़े & & & & 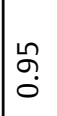 & & $\mid \begin{array}{c}0 \\
\vdots \\
0\end{array}$ & & & $\begin{array}{l}: \\
: \\
0 \\
0\end{array}$ & & & $\underset{\square}{\sigma}$ & & \\
\hline 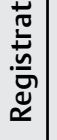 & $\begin{array}{l}0 \\
\vdots \\
\sim \\
\sim\end{array}$ & $\begin{array}{c}m \\
\stackrel{m}{0} \\
1\end{array}$ & $\begin{array}{l}n \\
0 \\
0 \\
0\end{array}$ & & $\mid \begin{array}{l}\sim \\
\stackrel{1}{1} \\
\stackrel{8}{8}\end{array}$ & $\begin{array}{l}\stackrel{2}{\Gamma} \\
+H \\
\infty \\
\infty\end{array}$ & & $\begin{array}{l}m \\
+ \\
8 \\
\sigma\end{array}$ & $\begin{array}{l}m \\
\frac{m}{H} \\
\frac{1}{\infty}\end{array}$ & $\begin{array}{l}+ \\
+ \\
+1 \\
\text { O }\end{array}$ & & $\begin{array}{l} \pm \\
+ \\
+ \\
\infty \\
\infty\end{array}$ & $\begin{array}{l} \pm \\
+ \\
+ \\
8 \\
\varnothing\end{array}$ & $\begin{array}{l}\infty \\
\stackrel{+}{+} \\
\infty \\
\infty\end{array}$ & & $\begin{array}{l}m \\
\stackrel{m}{H} \\
\delta \\
\sigma\end{array}$ & 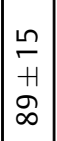 & $\begin{array}{l}0 \\
\stackrel{+}{H} \\
\dot{\sigma} \\
\sigma\end{array}$ & & $\begin{array}{l}m \\
\stackrel{m}{+} \\
\stackrel{2}{\infty}\end{array}$ & 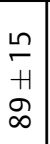 & $\begin{array}{l}\infty \\
H \\
\text { J }\end{array}$ & & & $\begin{array}{l}+ \\
+ \\
\stackrel{+}{ } \\
\infty\end{array}$ & 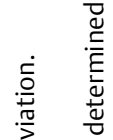 \\
\hline & 竞 & \begin{tabular}{|l|}
$n$ \\
$\infty$ \\
$\infty$ \\
0
\end{tabular} & $\begin{array}{l}9 \\
\stackrel{2}{0}\end{array}$ & & $\stackrel{m}{\check{0}}$ & & & 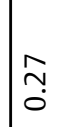 & & & & 号. & & & & 守 & & $\stackrel{\stackrel{n}{0}}{\check{0}}$ & & & $\begin{array}{c}\bar{\sigma} \\
0\end{array}$ & & & $\begin{array}{l}\frac{0}{2} \\
\vdots\end{array}$ & & 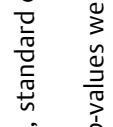 \\
\hline 竧 & 竞 & $\begin{array}{l}0 \\
0 \\
0\end{array}$ & $\begin{array}{l}8 \\
0 \\
0\end{array}$ & & $\mid \begin{array}{l}m \\
+ \\
+ \\
\infty \\
\infty\end{array}$ & $\mid \begin{array}{l}m \\
+ \\
+ \\
\infty \\
\infty\end{array}$ & & 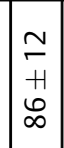 & $\begin{array}{l}\infty \\
+\frac{1}{1} \\
\infty\end{array}$ & $\begin{array}{l}\vec{J} \\
+ \\
+1 \\
\infty \\
\infty\end{array}$ & & $\begin{array}{l}m \\
\stackrel{m}{H} \\
\dot{H} \\
\infty\end{array}$ & $\mid \begin{array}{l}\mp \\
+ \\
\dot{8} \\
\sigma\end{array}$ & $\mid \begin{array}{l}\simeq \\
+1 \\
\sigma \\
\sigma\end{array}$ & & $\begin{array}{l}\mathfrak{N} \\
+ \\
\infty \\
\infty \\
\infty\end{array}$ & $\mid \begin{array}{l}\stackrel{n}{n} \\
+ \\
\stackrel{H}{n} \\
\infty\end{array}$ & $\mid \begin{array}{l}m \\
+ \\
\frac{1}{\infty}\end{array}$ & & $\begin{array}{l}\simeq \\
\stackrel{1}{+1} \\
\stackrel{2}{\infty}\end{array}$ & $\begin{array}{l}n \\
\stackrel{n}{+} \\
+ \\
\infty\end{array}$ & $\begin{array}{l}\simeq \\
+ \\
+ \\
\infty \\
\infty\end{array}$ & & $\begin{array}{l}\underset{m}{T} \\
+ \\
\dot{D}\end{array}$ & $\begin{array}{l}m \\
+ \\
+ \\
\infty \\
\infty\end{array}$ & 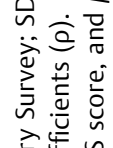 \\
\hline & 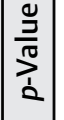 & $\begin{array}{l}\overline{6} \\
0 \\
\end{array}$ & $\mid \begin{array}{l}0 \\
2 \\
0\end{array}$ & & $\frac{m}{0}$ & & & $\stackrel{\widetilde{N}}{0}$ & & & & 帒 & & & & $\infty$ & & $\begin{array}{l}\infty \\
\stackrel{\infty}{0} \\
0\end{array}$ & & & $\begin{array}{c}\pi \\
0 \\
0\end{array}$ & & & $\frac{\pi}{\tilde{O}}$ & & $\left\{\begin{array}{l}0 \\
\frac{0}{0}\end{array}\right.$ \\
\hline $\begin{array}{l}\bar{\pi} \\
0 \\
\end{array}$ & 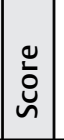 & \begin{tabular}{|c|} 
\\
0 \\
0 \\
0 \\
1
\end{tabular} & $\begin{array}{l}n \\
0 \\
0 \\
\end{array}$ & & $\begin{array}{l}0 \\
+ \\
\bar{H} \\
\sigma\end{array}$ & $\begin{array}{l}\sigma \\
+1 \\
\tilde{\sigma}\end{array}$ & & $\begin{array}{l}\infty \\
H \\
m \\
\sigma\end{array}$ & 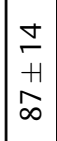 & $\begin{array}{l}\bar{\sigma} \\
+ \\
\sigma \\
\sigma\end{array}$ & & $\begin{array}{l}O \\
+ \\
\tilde{H} \\
\sigma\end{array}$ & $\begin{array}{l}\infty \\
+ \\
\dot{\sigma} \\
\end{array}$ & $\begin{array}{l}0 \\
+1 \\
0 \\
0\end{array}$ & & $\begin{array}{l}\sigma \\
H \\
\tilde{\sigma}\end{array}$ & $\begin{array}{l}\mp \\
+ \\
\sigma \\
\sigma\end{array}$ & $\begin{array}{l}\hat{y} \\
\dot{H} \\
\tilde{\sigma}\end{array}$ & & $\begin{array}{l}\sigma \\
+1 \\
m\end{array}$ & $\begin{array}{l}\bar{\sigma} \\
\bar{H} \\
\bar{\sigma}\end{array}$ & $\begin{array}{l}0 \\
+ \\
\text { Ln }\end{array}$ & & $\begin{array}{l}a \\
+ \\
m \\
\sigma\end{array}$ & $\begin{array}{l}\infty \\
+ \\
m \\
\sigma\end{array}$ & 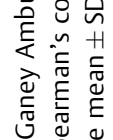 \\
\hline 总 & & 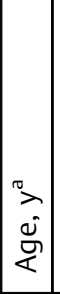 & 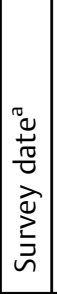 & $\mid \begin{array}{l}0 \\
\stackrel{x}{凶} \\
\backsim\end{array}$ & $\frac{0}{\frac{\pi}{N}}$ & 鸪 & 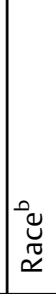 & 总 & 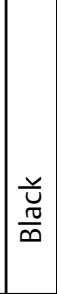 & 竞 & 气ֶ & 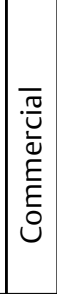 & 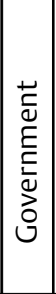 & 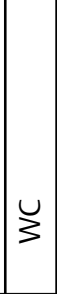 & \} $&{\text { 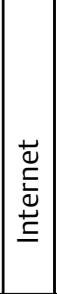 }} &{\text { 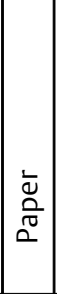 }} &{\text { 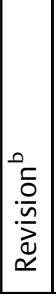 }} &{\text { 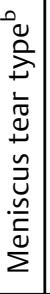 }} &{\text { 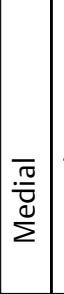 }} &{\text { 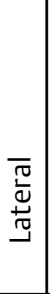 }} &{\text { 点 }} &{\text { 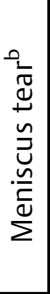 }} &{\text { 蛋 }} &{\begin{array}{l}0 \\
\tilde{z} \\
z \\
z\end{array}} &{\text { 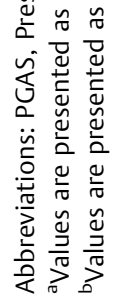 }} \\
$\hline
\end{tabular}




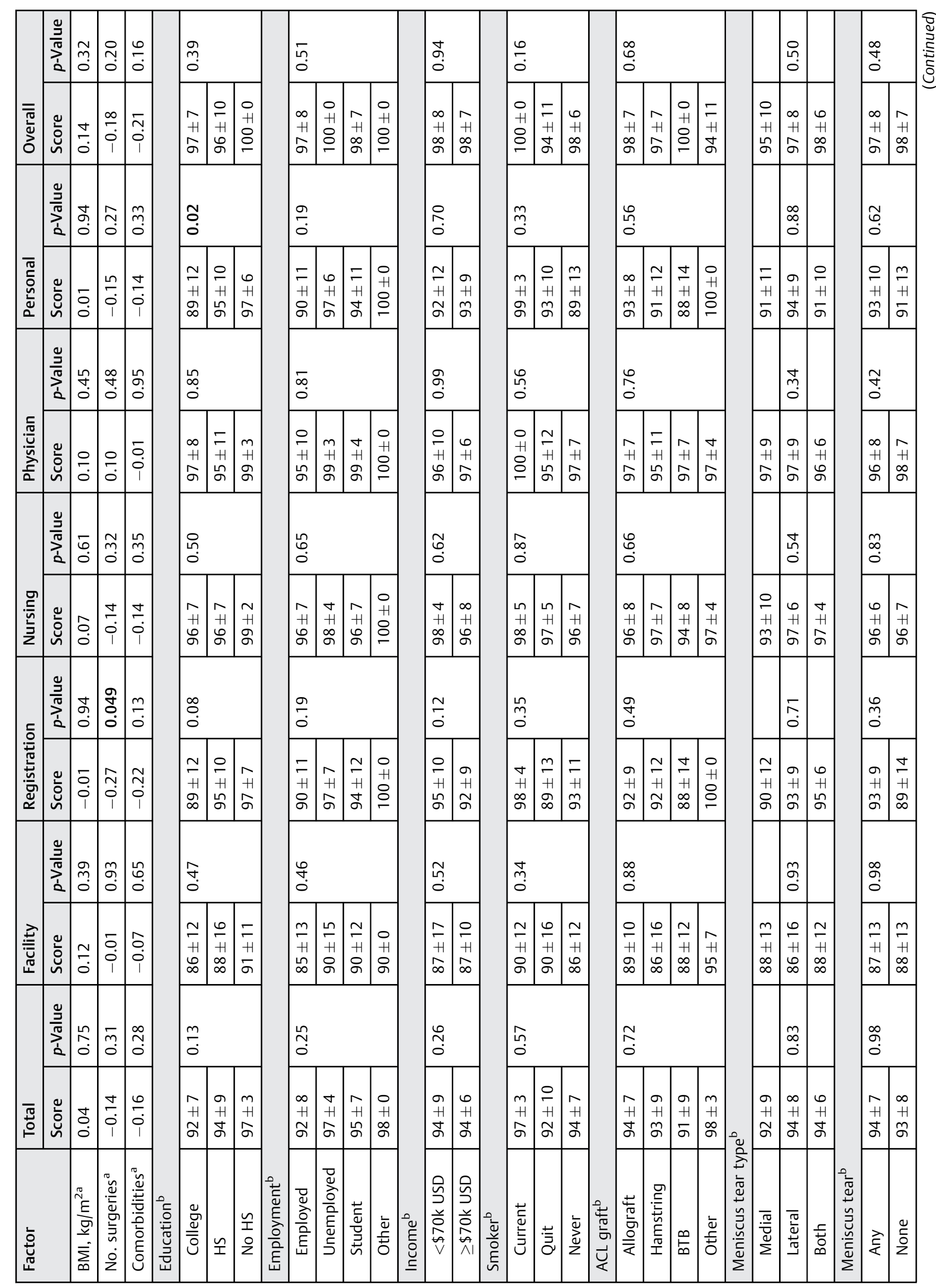




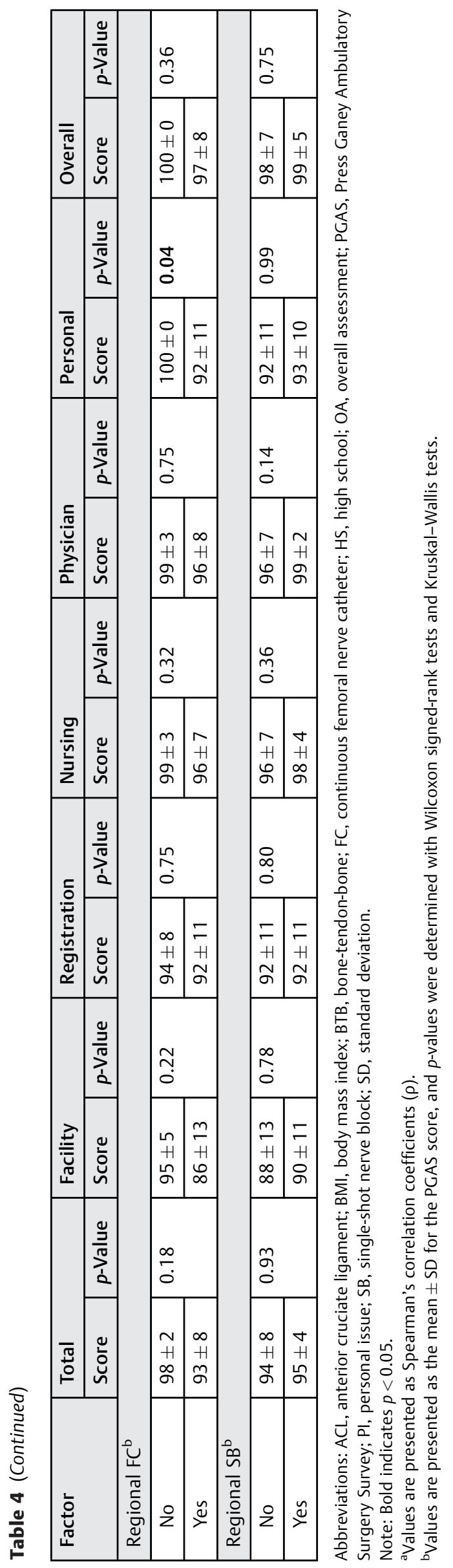

\section{Discussion}

Considering the emphasis healthcare has placed on patient satisfaction to determine physician reimbursement, understanding the factors affecting patients' perception of the operative experience is critical. There is a lack of prior research evaluating the factors associated with PG scores in sports medicine patients, particularly those undergoing ACLR. The current study identified several patient and surgical factors that were associated with worse PGAS domain scores in patients undergoing ACLR, but none of the factors were significantly associated with the total PGAS score. Additionally, the registration and facility domains were associated with the greatest variability in the total PGAS score, while the physician domain was the least influential. The results of this study do not provide reliable targets for hospital systems to improve total PGAS survey scores. The lack of correlation with validated baseline PROMs should caution the interpretation of PGAS scores and their use for future reimbursement.

Literature describing patient satisfaction following ACLR is limited, and no prior studies have determined the factors associated with PG survey scores in this patient population. Kocher et al previously determined predictors of patient satisfaction at a minimum of 2 years after ACLR, showing improved satisfaction with better 2-year IKDC scores and less satisfaction in the presence of a lateral meniscus tear. ${ }^{13}$ This is consistent with prior research demonstrating the longterm importance of the lateral meniscus. ${ }^{14,15}$ In contrast, the current study did not find an association between meniscus tears and PGAS scores.

Despite a lack of data reporting satisfaction after ACLR, satisfaction rates are generally high. A recent report demonstrated $85.4 \%$ of patients are very satisfied following ACLR, and $10.3 \%$ of patients are somewhat satisfied. ${ }^{16}$ These findings, however, were reported at a minimum of 2 years followup, and patients were more likely to be satisfied if they returned to sport. The current study showed similarly high satisfaction scores in the immediate postoperative period with a mean total PGAS score of 92 out of 100 points. Since PG has not released percentiles for PGAS scores after ACLR, the ceiling effects of the survey may render seemingly high scores in a low percentile nationally. ${ }^{17}$ Additionally, return to sport, a major contributor to patient satisfaction after ACLR, cannot be assessed within the 6 weeks postoperative timeframe when the PGAS survey is administered. Therefore, the relatively high total PGAS score reported in this study is unlikely to represent long-term patient satisfaction with their surgery, but rather satisfaction with their perioperative experience.

While no prior studies examined the association between PG surveys and PROMs in patients undergoing ACLR, multiple studies with conflicting results exist for other orthopaedic populations. For patients undergoing total hip and knee arthroplasty, Chughtai et al found no significant correlations between PG scores and common PROMs. ${ }^{1,6}$ These studies, however, assessed PROMs 2 years postoperatively, while the current study used baseline PROMs given their proximity to 


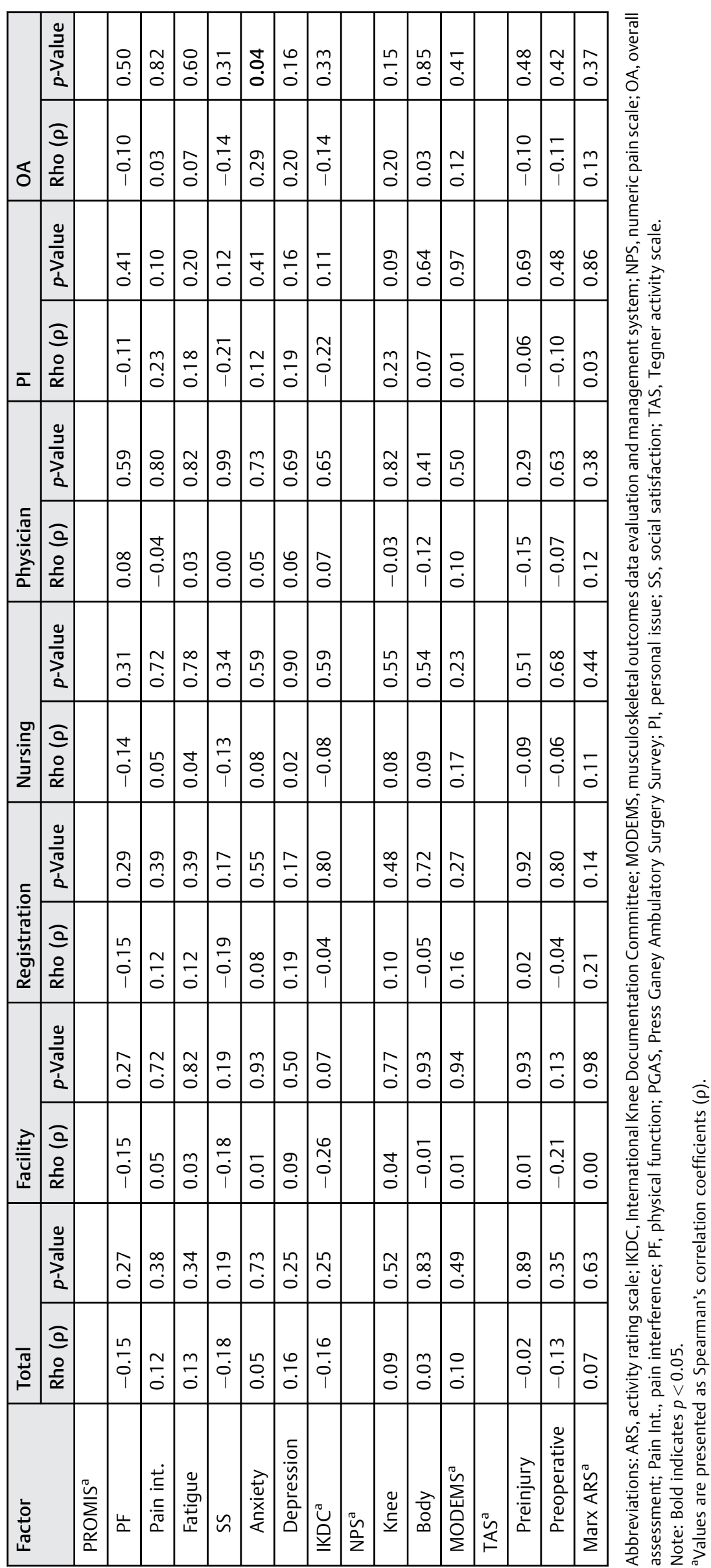


Table 6 Multivariable linear regression model for PGAS total score variability according to domain scores

\begin{tabular}{|l|l|l|l|l|l|l|}
\hline PGAS domain & Parameter estimate & $\beta$ (standardized) & SE & $p$-Value & VIF & ${\text { Adjusted } R^{2} \text { change (\%) }}$ \\
\hline Facility & 0.167 & 0.229 & 1.20 & $<0.001$ & 1.87 & 2.96 \\
\hline Registration & 0.167 & 0.238 & 0.01 & $<0.001$ & 1.85 & 3.07 \\
\hline Nursing & 0.167 & 0.215 & 0.01 & $<0.001$ & 3.21 & 1.51 \\
\hline Physician & 0.167 & 0.142 & 0.01 & $<0.001$ & 1.71 & 1.26 \\
\hline Personal issue & 0.167 & 0.226 & 0.02 & $<0.001$ & 3.59 & 1.50 \\
\hline Overall Assessment & 0.166 & 0.189 & 0.01 & $<0.001$ & 3.69 & 1.02 \\
\hline Adjusted $R^{2}$ & 0.999 & - & - & - & - & - \\
\hline
\end{tabular}

Abbreviations: PGAS, Press Ganey Ambulatory Surgery Survey; SE, standard error; VIF, variance inflation factor.

Note: Bold indicates $p<0.05$.

the timing of PGAS survey administration. The current study did not show any significant correlations between total PGAS and baseline PROMIS scores, but did show a significant, weakly positive correlation between PROMIS anxiety scores and the overall assessment domain. These findings conflict with prior research showing higher baseline PROMIS anxiety scores are associated with worse overall assessment PG scores in follow-up hand clinic patients. ${ }^{18}$ Inconsistent correlations between PROMs and PG surveys in orthopaedic research show the patient experience may be independent of measurable outcomes.

The results of this study showed correlations between several factors and PGAS domain scores. First, patients with a college degree had worse personal issue scores. By contrast, orthopaedic clinic patients with lower socioeconomic status have been previously shown to have worse PG scores. ${ }^{17}$ The findings in the present study may indicate patients with higher socioeconomic status have higher expectations of healthcare systems to properly address issues that arise during encounters. Second, patients who had a continuous femoral nerve catheter had worse personal issue scores, yet prior studies have shown pain control after total knee arthroplasty is associated with better patient satisfaction scores. ${ }^{9}$ These conflicting findings may be explained by the logistics of the continuous catheter itself, rather than the efficacy of pain control, as patients with or without a singleshot nerve block did not have significant differences in satisfaction scores. While the continuous femoral catheter is also a postoperative factor, it was included in this study because the catheter is placed preoperatively. Third, an increasing number of prior surgeries was weakly negatively correlated with registration PGAS scores. More surgical experiences likely provide greater context for the registration process, and deviation from patient expectations likely leads to worse scores in this domain. Finally, multiple authors in the outpatient clinic setting have shown that older age is associated with improved PG scores. ${ }^{3,8}$ The current study did not show associations between PGAS scores and older age, which is likely due to the younger cohort inherent to ACLR. Overall, the associations observed in the current study are weak, and the findings are likely not generalizable to other patient populations. It is important to recognize the inconsistent findings in the literature related to patient satisfaction surveys, as they potentially carry financial implications.

Finally, the registration and facility domain scores in the current study were responsible for the greatest variability in the total PGAS score. The physician domain accounted for the least variability in the total PGAS score and had the highest mean score of any domain. This indicates that the surgeons consistently met the expectations of patients during their encounters, and surgeons have little room to improve their PGAS scores. Much of the PGAS score variability remains outside of the control of the surgeon, despite one report that the physician score is the most influential for the total PG score in orthopaedic clinic patients. ${ }^{5}$ Such differences may be related to patient expectations in the clinic versus the surgical center, as patients are concerned with wait times and the time spent with the physician in the clinic setting. ${ }^{8,19}$

There are several limitations to this study. The greatest weakness of the study is the small sample size due to the $11.9 \%$ (99/829 ACLRs) response rate to the PGAS survey. The response rate is within the 8.9 to $16.5 \%$ range presented for orthopaedic clinic patients. ${ }^{8,20,21}$ The sample size in the current study is much smaller than prior PG studies, as most studies include heterogenous clinic populations. This study, however, specifically assessed ambulatory surgery encounters, which are less frequent than clinic encounters, in a homogenous population of patients undergoing ACLR over a 5-year period. Importantly, PG claims 30 responses is an adequate sample size to draw meaningful conclusions of a practice, and encounters with this minimum cutoff will be used for national rankings. ${ }^{4}$ So, the data in this study represent all the available responses for national rankings over a 5 -year period for a single institution. Second, a smaller cohort of patients $(n=54)$ was enrolled in the orthopaedic registry, limiting the analysis for certain variables. Differences in the subgroups were minimal, as only the number of meniscus tears were different in the registry group. Finally, the results of this study may not be generalizable to other patient populations given the data are from a single academic, urban population.

In summary, this is the first study to evaluate the PGAS survey in patients undergoing ACLR procedures. While no significant correlations were shown between preoperative patient factors and the total PGAS score, there were multiple weak correlations for the registration, personal issue, and 
overall assessment scores. Except for the type of regional anesthesia, the number of prior surgeries, education level, and baseline anxiety level of patients undergoing ACLR are largely nonmodifiable factors weakly associated with PGAS scores. Given these findings and a lack of consistency in the literature regarding the factors associated with PG scores, we cannot recommend specific modifiable patient targets for surgery centers to improve PGAS scores in patients undergoing ACLR. Given the registration and facility scores contributed the greatest variability to the total PGAS score, improving the patient experience in these areas may have the greatest impact. The surgeon contributed the least to the variability of the total score, and physician domain scores were consistently high, demonstrating that PGAS scores may be out of the control of the surgeon. This has important implications to future reimbursement and PGAS scores should be interpreted with caution. Future studies should determine if PGAS scores are correlated with surgical satisfaction and long-term PROMs in patients undergoing ACLR.

Funding

This work was supported in part by Career Development Award Number (grant no.: IK2 BX004879) from the United States (U.S.) Department of Veterans Affairs Biomedical Laboratory R\&D (BLRD) Service.

\section{Conflict of Interest}

J.D.P. reports educational support from Supreme Orthopedic Systems LLC, Smith \& Nephew Inc, and from Arthrex Inc outside the submitted work. S.J.M. reports educational support from Supreme Orthopedic Systems LLC, Smith \& Nephew Inc, Arthrex Inc, and Atlantic Surgical Systems LLC outside the submitted work. R.F.H. reports educational support and research funding from Arthrex Inc outside the submitted work. The rest of the authors declare no conflict of interest.

\section{Acknowledgments}

The authors thank J. Kathleen Tracy, PhD; Andrew G. Dubina, MD; Julio J. Jauregui, MD; Vidushan Nadarajah, MD; Craig H. Bennett, MD; Kenneth M. Chin, MD; Shaun H. Medina, MD; Cameran I. Burt; Dominic J. Ventimiglia; Keyan Shasti; Alexander J. Wahl; and Michael P. Smuda for their assistance with data collection.

\section{References}

1 Chughtai M, Patel NK, Gwam CU, et al. Do Press Ganey scores correlate with total knee arthroplasty-specific outcome questionnaires in postsurgical patients? J Arthroplasty 2017;32(9S): S109-S112

2 Etier BE Jr, Orr SP, Antonetti J, Thomas SB, Theiss SM. Factors impacting Press Ganey patient satisfaction scores in orthopedic surgery spine clinic. Spine J 2016;16(11):1285-1289

3 Abtahi AM, Presson AP, Zhang C, Saltzman CL, Tyser AR. Association between orthopaedic outpatient satisfaction and non-modifiable patient factors. J Bone Joint Surg Am 2015;97(13):1041-1048
4 Zusman EE. HCAHPS replaces Press Ganey survey as quality measure for patient hospital experience. Neurosurgery 2012;71 (02):N21-N24

5 Martinez JR, Nakonezny PA, Batty M, Wells J. The dimension of the Press Ganey survey most important in evaluating patient satisfaction in the academic outpatient orthopedic surgery setting. Orthopedics 2019;42(04):198-204

6 Chughtai M, Gwam CU, Khlopas A, et al. No correlation between Press Ganey survey responses and outcomes in post-total hip arthroplasty patients. J Arthroplasty 2018;33(03):783-785

7 Kohring JM, Pelt CE, Anderson MB, Peters CL, Gililland JM. Press Ganey outpatient medical practice survey scores do not correlate with patient-reported outcomes after primary joint arthroplasty. J Arthroplasty 2018;33(08):2417-2422

8 Rane AA, Tyser AR, Presson AP, Zhang C, Kazmers NH. Patient satisfaction in the hand surgery clinic: an analysis of factors that impact the Press Ganey survey. J Hand Surg Am 2019;44(07): 539-547.e1, e531

9 Chughtai M, Jauregui JJ, Mistry JB, et al. What influences how patients rate their hospital after total knee arthroplasty? Surg Technol Int 2016;28:261-265

10 Ganey P. OAS CAHPS FAQ. Accessed December 5, 2021 at: https:// www.pressganey.com/resources/program-summary/oas-cahpsfaq. Published 2019.

11 Harris PA, Taylor R, Thielke R, Payne J, Gonzalez N, Conde JG. Research electronic data capture (REDCap)-a metadata-driven methodology and workflow process for providing translational research informatics support. J Biomed Inform 2009;42(02): 377-381

12 Henn RF III, Dubina AG, Jauregui JJ, Smuda MP, Tracy JK. The Maryland Orthopaedic Registry (MOR): design and baseline characteristics of a prospective registry. J Clin Orthop Trauma 2017;8 (04):301-307

13 Kocher MS, Steadman JR, Briggs K, Zurakowski D, Sterett WI, Hawkins RJ. Determinants of patient satisfaction with outcome after anterior cruciate ligament reconstruction. J Bone Joint Surg Am 2002;84(09):1560-1572

14 Allen PR, Denham RA, Swan AV. Late degenerative changes after meniscectomy. Factors affecting the knee after operation. J Bone Joint Surg Br 1984;66(05):666-671

15 Jones MH, Spindler KP, Andrish JT, et al; MOON Knee Group. Differences in the lateral compartment joint space width after anterior cruciate ligament reconstruction: data from the MOON onsite cohort. Am J Sports Med 2018;46(04):876-882

16 Nwachukwu BU, Voleti PB, Berkanish P, et al. Return to play and patient satisfaction after ACL reconstruction: study with minimum 2-year follow-up. J Bone Joint Surg Am 2017;99(09): 720-725

17 Stephens AR, Tyser AR, Kazmers NH. The impact of social deprivation on orthopaedic outpatient satisfaction using the Press Ganey Outpatient Medical Practice Survey. J Am Acad Orthop Surg 2020;28(24):e1111-e1120

18 Tyser AR, Gaffney CJ, Zhang C, Presson AP. The association of patient satisfaction with pain, anxiety, and self-reported physical function. J Bone Joint Surg Am 2018;100(21):1811-1818

19 Rane AA, Tyser AR, Kazmers NH. Evaluating the impact of wait time on orthopaedic outpatient satisfaction using the Press Ganey survey. JBJS Open Access 2019;4(04):e0014

20 Compton J, Glass N, Fowler T. Evidence of selection bias and nonresponse bias in patient satisfaction surveys. Iowa Orthop J 2019; 39(01):195-201

21 Tyser AR, Abtahi AM, McFadden M, Presson AP. Evidence of nonresponse bias in the Press-Ganey patient satisfaction survey. BMC Health Serv Res 2016;16(a):350 